\title{
Ultraviolet Emission from the LINER Nucleus of NGC 6500円
}

\author{
Aaron J. Barth ${ }^{2}$, Gail A. Reichert ${ }^{3}$, Luis C. Ho ${ }^{4}$, Joseph C. Shields ${ }^{5}$, Alexei V. Filippenko ${ }^{2}$, \\ and Elizabeth M. Puchnarewicz ${ }^{6}$
}

\begin{abstract}
As a step towards clarifying the ionization mechanism of LINERs, we have used the Hubble Space Telescope Faint Object Spectrograph to obtain an ultraviolet spectrum of the nucleus of NGC 6500. This is the first time that such a spectrum has been taken of a "LINER 2" nucleus- that is, a LINER lacking broad permitted emission lines like those in Type 1 Seyfert nuclei. Compared with more luminous Seyfert nuclei, the ultraviolet emission-line spectrum of NGC 6500 is remarkable for its low-excitation character: C III] $\lambda 1909, \mathrm{C}$ II] $\lambda 2326$, and $\mathrm{Mg}$ II $\lambda 2800$ are the strongest collisionallyexcited lines in the spectrum, and C IV $\lambda 1549$ is not detected. We use the emissionline fluxes to test the hypothesis, advanced by Dopita \& Sutherland (1995), that the narrow-line regions of LINERs are primarily excited by fast $\left(150-500 \mathrm{~km} \mathrm{~s}^{-1}\right)$ shocks, rather than photoionized by a central continuum source. The fast shock models are a poor match to the observed spectrum, as they predict much stronger emission than is observed for high-excitation lines such as C IV $\lambda 1549$. Photoionization by an obscured nonstellar continuum source, or possibly ionization by slower $\left(\sim 100 \mathrm{~km} \mathrm{~s}^{-1}\right)$ shocks, are more likely explanations for the emission-line ratios. The origin of the ultraviolet continuum is unclear; its overall spectral shape is reasonably well matched by spectral evolution models for single-burst populations with ages in the range 70-100 Myr, but no definite stellar absorption features due to young stars are present. Alternately, the ultraviolet continuum (or some fraction of it) may be scattered radiation from a hidden active nucleus. We tentatively detect a broad He II $\lambda 4686$ emission feature, which may be due to Wolf-Rayet stars, although the ultraviolet spectrum does not show any clear Wolf-Rayet signatures. We also find that NGC 6500 is weakly detected in an archival
\end{abstract}

\footnotetext{
${ }^{1}$ Based on observations made with the NASA/ESA Hubble Space Telescope, obtained from the Space Telescope Science Institute, which is operated by the Association of Universities for Research in Astronomy, Inc., under NASA contract NAS 5-26555.

${ }^{2}$ Department of Astronomy, University of California, Berkeley, CA 94720-3411.

${ }^{3}$ Code 668, NASA/Goddard Space Flight Center, Greenbelt, MD 20771.

${ }^{4}$ Harvard-Smithsonian Center for Astrophysics, Cambridge, MA 02138.

${ }^{5}$ Ohio University, Physics and Astronomy Department, Athens OH, 45701.

${ }^{6}$ Mullard Space Science Laboratory, University College London, Holmbury St. Mary, Dorking, Surrey RH5 6NT, UK.
} 
ROSAT HRI image with a luminosity of $\sim 5 \times 10^{40} \mathrm{erg} \mathrm{s}^{-1}$ in the $0.1-2.4 \mathrm{keV}$ band, within the normal range for LINERs.

\section{Introduction}

Low-ionization nuclear emission-line regions, or LINERs, occur in one-third of all bright, nearby galaxies and in nearly 50\% of early-type (E-Sab) galaxies (Heckman 1980; Ho et al. 1997a), yet in most cases the physical mechanism responsible for the ionization is not well understood]. It is likely that LINERs comprise a heterogeneous class in which stellar and nonstellar photoionization, as well as shocks, contribute in varying degrees to the energetics of individual objects. The presence of broad $\mathrm{H} \alpha$ emission in approximately $20 \%$ of LINERs (Ho et al. 1997b) is a clear indication of a link with more luminous active galactic nuclei (AGNs), and one can define "LINER 1" and "LINER 2" subclasses, based on the presence or absence of broad $\mathrm{H} \alpha$ emission, in a direct analogy to the Seyfert galaxy population. The status of the more numerous LINER 2s is less clear, however. Some fraction of these objects may be obscured LINER 1 nuclei, or low-luminosity AGNs which simply lack a luminous broad-line region (BLR). Nevertheless, shock excitation and photoionization by young, massive stars are viable alternatives for the ionization mechanism of the narrow-line gas in these objects (see Filippenko 1996 for a review). If LINER 2 nuclei are indeed a subclass of the AGN family, then they are the most common form of AGN to occur in nearby galaxies. It is therefore of great importance to clarify their nature if we are to develop a complete understanding of the AGN phenomenon at low luminosities and of the interstellar medium in the central regions of early-type galaxies.

Recently, Dopita \& Sutherland (1995, 1996; hereafter DS) have computed new grids of fast (150-500 $\mathrm{km} \mathrm{s}^{-1}$ ) shock models, and have shown that the emission-line spectra of fast shocks can match the range of optical line ratios observed in Seyfert nuclei and LINERs. Based on this result, they propose that the narrow-line regions (NLRs) of all AGNs are primarily shock-excited, rather than photoionized by the central continuum source. For a critical discussion of the DS fast shock models, see Morse et al. (1996). Optical emission-line diagnostics for Seyferts and LINERs do not yield a definitive test of the shock hypothesis, but one area in which the shock models disagree most strongly with standard photoionization models is in their predictions for the UV spectra of LINERs. Because of the high temperatures generated by the passage of a shock front, the fast shock models predict far stronger emission in high-excitation UV lines than do traditional photoionization models for LINERs. Thus far, however, only three HST UV spectra of LINERs have been published: the LINER 1 nuclei of M81 (Ho et al. 1996) and NGC 4579 (Barth et al. 1996b) have UV spectra which are more consistent with the predictions of photoionization models than with the fast shock models, while an off-nuclear spectrum of M87 (Dopita et al. 1996) reveals high-excitation emission

\footnotetext{
${ }^{7}$ As defined by Heckman (1980), a LINER has [O II] $\lambda 3727 /[\mathrm{O}$ III] $\lambda 5007 \geq 1$ and $[\mathrm{O}$ I] $\lambda 6300 /[\mathrm{O}$ III] $\lambda 5007$ $\geq 1 / 3$.
} 
which may be more consistent with the shock hypothesis.

NGC 6500 presents a particularly interesting target for such observations. This galaxy (type Sab; $c z=3003 \mathrm{~km} \mathrm{~s}^{-1}$ ) is a well-known LINER 2 with strong emission lines (e.g., Filippenko \& Sargent 1985). Radio observations reveal the presence of a compact flat-spectrum nuclear source (Crane 1979; Jones et al. 1981) as well as extended lobes of emission emerging perpendicular to the galactic major axis (Unger et al. 1989). The lobes appear to be the signature of a nuclear outflow or wind, and the ionized gas shows kinematic signatures of an outflow as well González Delgado \& Pérez 1996). A diffuse $\sim 100$ pc region of UV emission at the nucleus (Barth et al. 1996a) suggests that a nuclear starburst could be responsible for the outflow, and the possible presence of WolfRayet (WR) spectral features in the nuclear spectrum, as we discuss in $\S 3.1$ below, reveals that star formation may have occurred within the last few Myr as well. In the circumnuclear region, low-ionization emission is seen out to radii of $\sim 1 \mathrm{kpc}$ (see the off-nuclear spectra presented by Filippenko 1984 and González Delgado \& Pérez 1996), unlike the case in the majority of LINERs where the emission is largely confined to the inner few hundred pc (Pogge 1989). This evidence for a nuclear outflow makes NGC 6500 an ideal object with which to test the predictions of shock models for LINERs. In this paper, we present HST UV spectra of the nucleus of NGC 6500, and apply our results to a test of the fast shock hypothesis. This is the first LINER 2 nucleus for which such observations have been obtained. It has been argued (Dopita et al. 1996) that the more Seyfert-like LINER 1 nucleus of M81 is not a valid testing ground for the shock models because of the somewhat higher excitation level of its optical emission lines, but NGC 6500 is classified as a bona fide LINER (González Delgado \& Pérez 1996; Ho et al. 1997d) and such criticisms should not apply to it. Throughout this paper we assume a Hubble constant of $H_{0}=65 \mathrm{~km} \mathrm{~s}^{-1} \mathrm{Mpc}^{-1}$, corresponding to a distance of $46 \mathrm{Mpc}$ to NGC 6500.

\section{Observations and Measurements}

NGC 6500 was observed by the post-refurbishment HST Faint Object Spectrograph (FOS) on 1994 August 13 UT through the 0!'86-diameter circular aperture. A three-stage peakup with an expected accuracy of 0 ' 12 was used to center the aperture on the optical nucleus of the galaxy. Three grating/detector combinations were used, covering the range 1160-3270 A: the G130H grating with the FOS/BLUE detector $\left(1.0 \AA\right.$ diode $\left.^{-1}\right)$, and the G190H $\left(1.5 \AA\right.$ diode $\left.^{-1}\right)$ and G270H $(2.1 \AA$ diode $^{-1}$ ) gratings with the FOS/RED detector. Total exposure times were 10260, 3110, and 1020 $\mathrm{s}$, respectively. The point source spectral resolution in each setting is 0.96 diode, corresponding to $220 \mathrm{~km} \mathrm{~s}^{-1}$, while a source uniformly filling the aperture would have a spectral resolution of 520 $\mathrm{km} \mathrm{s}^{-1}$.

The data were processed by the routine HST calibration pipeline, which includes flat-fielding, subtraction of the particle-induced background, and flux and wavelength calibration. For each grating setting, we co-added and rebinned the spectra to a wavelength bin size corresponding to the width of one diode. The calibrated spectra are shown in Figure 1. From the emission-line peaks, 
we find that small offsets occur between the wavelength scales of the three gratings (see Koratkar $\&$ Martin 1995 for a discussion of FOS wavelength inaccuracies), and the spectra in Figure 1 are displayed after shifting to a common wavelength scale and removing the galaxy's recession velocity.

The FOS scattered light problem (e.g., Rosa 1994), which primarily affects the G130H setting, is particularly severe for these data because of the extremely faint UV continuum of NGC 6500. By examination of the uncalibrated $\mathrm{G} 130 \mathrm{H}$ data frames in the unilluminated regions shortward of 1150 $\AA$, we estimate that $\sim 25 \%$ of the total counts at $1300 \AA$ are due to scattered light, while another $\sim 50 \%$ are due to particle-induced background light. The standard calibration pipeline corrects for these two sources of contamination, but the scattered light correction is only approximate, as the distribution of scattered light over the array is not well known. The spectral shape of the continuum is drastically altered by the correction, and we cannot reliably recover the spectral shape below $1600 \AA$. The scattered light correction does not affect the strengths of emission features, however.

The G190H and G270H spectra have much higher count rates and should not be strongly affected by scattered light, but in these settings there are no unilluminated diodes that can be used to estimate the scattered light contribution. In the overlap region between the G190H and G270H settings (observed wavelength 2224-2310 $\AA$ ), there is an upturn in the continuum in the G190H spectrum which does not appear in the G270H spectrum, and the flux level in $\mathrm{G} 190 \mathrm{H}$ is $20 \%$ greater than in $\mathrm{G} 270 \mathrm{H}$ over this wavelength range. To construct the complete spectrum, we took the mean of the two settings in this region. As a consistency check on the flux scale, we compared our data with the count rate measured in a WFPC2 F218W image of NGC 6500 (Barth et al. 1996a), using the SYNPHOT package within IRAF to measure the F218W magnitude of the FOS spectrum. We find that the FOS-derived flux is $33 \%$ greater than the flux level of the WFPC2 image; the cause of this discrepancy is unknown.

To measure the UV emission-line strengths, we first removed the continuum around each line by fitting a low-order spline to the local continuum and subtracting the spline from the data. Line intensities were then measured by direct integration of the flux, and, when possible, by Gaussian fits. Table 1 lists the measured emission-line fluxes. The uncertainty in the placement of the continuum level is a major source of error for He II $\lambda 1640$, which lies at the very noisy blue end of the G190H spectrum. Neglecting calibration errors, the uncertainties in the emission-line fluxes are roughly $\sim 15 \%$ for the stronger lines but are probably $\sim 30-50 \%$ for He II $\lambda 1640$, Si III $\lambda \lambda 1882$, and $[\mathrm{O}$ II $] \lambda 2470$. The line intensities are not corrected for the effects of interstellar absorption, which is clearly significant for $\mathrm{Mg}$ II $\lambda 2800$ and possibly also for Ly $\alpha$.

The full-width at half-maximum (FWHM) was measured by Gaussian fitting, but meaningful results were obtained only for Ly $\alpha, \mathrm{C}$ III] $\lambda 1909$, and C II] $\lambda 2326$. Instrumental broadening was subtracted in quadrature from the line widths using the extended-source resolution of $520 \mathrm{~km} \mathrm{~s}^{-1}$,

\footnotetext{
${ }^{8}$ IRAF is distributed by the National Optical Astronomy Observatories, which are operated by the Association of Universities for Research in Astronomy, Inc., under cooperative agreement with the National Science Foundation.
} 
as the nuclear emission-line region of NGC 6500 is probably better approximated by a uniform source than a pointlike source on $1^{\prime \prime}$ scales.

Because of the limited signal-to-noise ratio $(\mathrm{S} / \mathrm{N})$ of the spectrum, a few lines are contaminated by noisy diodes or have ambiguous identifications. A noisy diode may contribute to the apparent flux of the [O II] $\lambda 2470$ line; we have attempted to measure the true line flux by manually truncating the blue half of the line profile to match the red half, but the resulting flux is probably uncertain by $\sim 50 \%$. A noisy diode also appears at the expected position of N II] $\lambda 2140$ and renders impossible the detection of that line. A weak emission feature of formal significance $2 \sigma$ appears near the expected location of $\mathrm{N} \mathrm{V} \lambda 1240$, but it may simply be a peak in the noise, particularly because other high-excitation lines such as C IV $\lambda 1549$ and O IV $\lambda 1402$ are not detected in the G130H spectrum. We therefore interpret the possible N V measurement as an upper limit. Two emission features are present at rest wavelengths 2611 and $2626 \AA$. These wavelengths are coincident with those of two transitions in the UV1 multiplet of Fe II, but it is also possible that the features are due to noisy diodes.

The main result of this paper is the non-detection of C IV $\lambda 1549$ emission. We determined a $3 \sigma$ upper limit to the flux of C IV of $1.0 \times 10^{-15} \mathrm{erg} \mathrm{s}^{-1} \mathrm{~cm}^{-2}$ by examining the continuum noise in the region 1500-1600 A. The FWHM of C IV was assumed to be $1200 \mathrm{~km} \mathrm{~s}^{-1}$, since C IV is likely to be at least as broad as C III] $\lambda 1909$.

Optical emission-line fluxes measured from starlight-subtracted spectra have been given by Ho et al. (1997c). Since the ground-based spectra were obtained through a $2^{\prime \prime} \times 4^{\prime \prime}$ effective aperture, we cannot directly compare the UV and optical emission-line strengths.

NGC 6500 lies at a Galactic latitude of $20^{\circ}$, and its spectrum is affected by a substantial amount of extinction. Ho et al. (1997c) measure a Balmer decrement of $\mathrm{H} \alpha / \mathrm{H} \beta=3.64$ for NGC 6500. Assuming an intrinsic Balmer decrement of 3.1, which is a typical value for the shock and photoionization models discussed below, we estimate a total reddening of $E(B-V)=0.16$ mag for the nucleus of NGC 6500. The Galactic H I column toward NGC 6500 is $7.0 \times 10^{20} \mathrm{~cm}^{-2}$ (Murphy et al. 1996). Using the conversion factor given by Mathis (1990), this H I column implies that the Galactic contribution to $E(B-V)$ is $0.12 \mathrm{mag}$, somewhat higher than the estimate of $E(B-V)=0.09 \mathrm{mag}$ given by Burstein \& Heiles (1984). Extinction-corrected line intensities, computed using the extinction curve of Cardelli et al. (1989), are listed in Table 1. It is important to bear in mind that the reddening curve within the NGC 6500 nucleus may be very different from the Galactic law, but Galactic dust is the dominant contribution to the reddening of this object, and in any case the reddening correction has less than a $10 \%$ effect on our primary diagnostic of the NLR ionization level, the C III]/C IV flux ratio.

An archival ROSAT HRI (0.1-2.4 keV) image taken on date 1993 March 17 shows that NGC 6500 is detected at the $3 \sigma$ level in a $5954 \mathrm{~s}$ exposure, with a total of $15 \pm 5$ counts above background within a square aperture of size $24^{\prime \prime} \times 24^{\prime \prime}$. Using the energy-to-count rate conversion data in the ROSAT HRI Calibration Report (David et al. 1996), and assuming an $f_{\nu} \propto \nu^{-1}$ continuum and the 
Galactic H I column given by Murphy et al. (1996), we find an unabsorbed source flux of $2 \times 10^{-13}$ erg $\mathrm{cm}^{-2} \mathrm{~s}^{-1}$. This flux corresponds to an X-ray luminosity of $5 \times 10^{40} \mathrm{erg} \mathrm{s}^{-1}$ in the $0.1-2.4 \mathrm{keV}$ band, which is within the normal range for LINERs (see Fabbiano 1996 and Serlemitsos et al. 1996) and superwind galaxies (Heckman et al. 1990).

\section{Discussion}

\subsection{The UV Continuum}

The region emitting the UV continuum is diffuse, with a diameter of $\sim 100 \mathrm{pc}$, and it lacks a central peak at the location of the optical nucleus (Barth et al. 1996a). By subtracting model point-spread functions from the WFPC2 image, we estimate that at most $\sim 7 \%$ of the $2200 \AA$ flux could be due to a single point source. Thus, an unobscured AGN could make only a very small contribution to the UV continuum. The two most likely sources of the extended UV emission are starlight and scattered radiation from a hidden AGN, and we discuss these possibilities below.

Correction of the continuum shape for the total reddening of $E(B-V)=0.16$ mag using a Galactic extinction law leads to an unacceptably large bump at $2200 \AA$ in the corrected spectrum. Even correcting for $E(B-V)=0.12 \mathrm{mag}$, the extinction inferred from the Galactic H I column, leads to a distinct rise in the continuum at $2200 \AA$. Using the Burstein \& Heiles (1984) value of $E(B-V)=0.09 \mathrm{mag}$ does not result in a noticeable $2200 \AA$ bump. The dangers of applying a Galactic extinction law to extragalactic starburst regions have been discussed by Calzetti et al. (1994), who conclude that the internal extinction law within starbursts is flatter than the Galactic law and lacks the $2200 \AA$ feature, and also that the optical continua of starbursts are less reddened than the emission lines. Since it is not clear how to best correct the UV continuum of NGC 6500 for the effects of extinction, particularly for the internal component, we choose to correct the UV continuum only for $E(B-V)=0.09$ mag using the Galactic extinction law, and we warn that we may have not fully removed the effects of extinction from the continuum.

An upturn in the continuum longward of $2800 \AA$ can plausibly be attributed to the increasing contribution of late-type stars at these wavelengths. An absorption feature at the expected location of the $3096 \AA$ blend adds support to this interpretation, as this feature (a blend of Fe I lines and Al I $\lambda$ 3093) appears in stellar spectra of type F7 and later (Fanelli et al. 1990). In the remainder of this section we address the question of the origin of the continuum flux shortward of $2800 \AA$.

Due to the low $\mathrm{S} / \mathrm{N}$ in the continuum, there are no definite stellar absorption features that can be identified with known features in stellar UV spectra. A broad depression of equivalent width (EW) $\sim 4 \AA$ appears in the region 1536-1545 $\AA$ (rest wavelength), and may be blueshifted C IV stellar-wind absorption. A spectrum with higher $\mathrm{S} / \mathrm{N}$ would be necessary to confirm the reality of this feature, and we interpret the measured EW of $4 \AA$ as an upper limit rather than a detection. We place an upper limit of $2 \AA$ on the EW of any Si IV $\lambda 1400$ absorption. The only definite 
absorption features in the spectrum are the Galactic and internal interstellar absorption features due to the UV2 multiplet of Fe II (2586 and $2599 \AA)$ and the Mg II doublet, which appear in the G270H spectrum.

The shape of the UV continuum is roughly similar to those of archival International Ultraviolet Explorer (IUE) spectra of late B-type and early A-type stars, suggesting that the UV continuum may originate in a young stellar population. To determine the age of the stellar population that most closely matches the observed spectrum, we compare the data with synthetic spectra generated by the isochrone synthesis code GISSEL96 (Bruzual \& Charlot 1993). Figure 2 shows the UV continuum shape of NGC 6500 and the spectra predicted for single-burst models of solar metallicity and a Salpeter (1955) initial mass function (IMF), for three different burst ages. The overall spectral shape is bracketed by burst ages ranging from $36 \mathrm{Myr}$ to $130 \mathrm{Myr}$, with the best overall match to the data for ages of 70-100 Myr. Because of the large uncertainty in the continuum shape below $1600 \AA$, there is little information in the spectrum that can distinguish the single-burst models from models with more complex star-formation histories, and we restrict our attention to the single-burst models. The model spectra with $t \gtrsim 130 \mathrm{Myr}$ are clearly too red to match the data, allowing us to set an upper age limit of $130 \mathrm{Myr}$ for the stellar population responsible for the bulk of the UV emission. In the wavelength range 1300-2300 $\AA$, the 36 Myr burst has a slope roughly similar to that of NGC 6500, and because of the uncertainties in the reddening and in the continuum shape below $1600 \AA$, we cannot rule out a substantial contribution from even younger stars. No single-burst model can fit the entire UV spectral region, however, as the upturn longward of $2800 \AA$ indicates the presence of an older population of stars. An excess over the model spectra is present at 2300-2500 $\AA$, partly due to a few sharp "bumps" in the region between C II] $\lambda 2326$ and [O II] $\lambda 2470$, and we are unable to explain this excess in terms of any normal stellar population.

To what extent might this young stellar population be responsible for the NLR excitation? Shields (1992; see also Filippenko \& Terlevich 1992) has shown that early O-type stars can give rise to a LINER-like optical spectrum if the surrounding interstellar medium is sufficiently dense. The possible presence of WR emission in the optical spectrum (see below) indicates that stars may have been forming within the last few Myr, and the possible blueshifted C IV absorption may be a further indication of stars with $M>30 M_{\odot}$ (Leitherer et al. 1995). As a rough comparison with our upper limits to the strength of C IV and Si IV absorption in NGC 6500 (4 $\AA$ and $2 \AA$, respectively), we note that the brightest starburst knot in NGC 4214 , which has an age of $4-5 \mathrm{Myr}$, has $\mathrm{EW}(\mathrm{C}$ IV $)=5.9 \AA$ and $\mathrm{EW}(\mathrm{Si} \mathrm{IV})=3.9 \AA$ (Leitherer et al. 1996). Thus, if a similarly young population were present in NGC 6500, its $1500 \AA$ continuum would likely be diluted by an equal or greater contribution from B and A stars, or by scattered AGN continuum radiation.

If the NLR luminosity were solely due to ionization by massive stars in the nucleus, then the ionizing photon luminosity of the nuclear starburst region must be at least $2.7 \times 10^{52} \mathrm{~s}^{-1}$ to account for the nuclear $\mathrm{H} \beta$ flux, assuming Case $\mathrm{B}$ recombination. To obtain a hard upper limit to the possible ionizing photon luminosity due to stars in the nucleus, we assume that the 1500 $\AA$ continuum flux arises entirely from a young starburst with age $<5 \mathrm{Myr}$, and we apply the full 
extinction correction of $E(B-V)=0.16$ mag to this flux. Using the results of Leitherer \& Heckman (1995) and Leitherer et al. (1995) for a solar-metallicity instantaneous burst with a power-law IMF of slope -2.35 and upper mass cutoff $100 M_{\odot}$, we find that the extinction-corrected $1500 \AA$ flux translates to a population of $\sim 10^{3}$ O-type stars and an ionizing photon luminosity of $2.0 \times 10^{52}$ $\mathrm{s}^{-1}$, or $74 \%$ of the amount required to ionize the NLR. However, the true ionizing photon output from hot stars is likely to be far less than this figure, because the apparent weakness of C IV and Si IV absorption imply that the majority of the $1500 \AA$ flux is not likely to come from O-type stars, and because the extinction correction of $E(B-V)=0.16$ mag is likely to be an overcorrection. We cannot rule out a sizeable contribution by hot stars to the NLR energetics, but it seems unlikely that hot stars could be the dominant contribution.

The possible detection of an optical He II $\lambda 4686$ feature provides supporting evidence for a recent burst of star formation. Figure 3 shows the starlight-subtracted optical spectrum of NGC 6500 (from Ho et al. 1997c), illustrating the 4600-4700 $\AA$ feature, which we tentatively attribute to WR stars. Its profile appears similar to the WR feature found in NGC 3049 (Vacca \& Conti 1992): He II $\lambda 4686$ is clearly present, nebular [Fe III] $\lambda 4658$ emission is superposed on the WR emission complex, and C IV $\lambda 4658$ from WC stars may be present as well. There may be weak emission from N III $\lambda 4640$, but the feature at this wavelength does not stand out above the noise level. NGC 6500 differs from most WR galaxies in that its nuclear optical continuum is dominated by old stars; most known WR galaxies have a relatively featureless, blue optical continuum due to massive stars. In NGC 6500, the WR emission blend only becomes visible after subtraction of the underlying starlight continuum. The width of the He II emission line adds support to the WR interpretation: a single-Gaussian fit to He II yields FWHM $\approx 1200 \mathrm{~km} \mathrm{~s}^{-1}$, while the strong optical emission lines have FWHM $=550-700 \mathrm{~km} \mathrm{~s}^{-1}$ (Ho et al. 1997d). Taking care not to include the adjacent [Fe III] $\lambda 4658$ emission, we find a flux of $2.5 \times 10^{-15} \mathrm{erg} \mathrm{s}^{-1} \mathrm{~cm}^{-2}$ for the dereddened He II line. Compared with the noise level in the region 3280-4630 $\AA$, the significance of the He II detection is $2.6 \sigma$. The true significance of the detection must be lower than this value, because of the unknown systematic uncertainties involved in the starlight subtraction as well as the possible nebular contribution to the $\lambda 4686$ feature.

Using the calibrations given by Vacca \& Conti (1992), the strength of the He II $\lambda 4686$ WR emission (less the unknown nebular contribution) implies the presence of $<350 \mathrm{WN}$ stars. Depending on the age and metal abundance of the young stellar population, the WR/O star ratio can vary from $\lesssim 0.1$ to $>1$ (Leitherer \& Heckman 1995), so the WR census is at least consistent with the upper limit on the O-star population given above. Optical spectra from the Space Telescope Imaging Spectrograph (STIS) could resolve much of the ambiguity in these estimates, as the narrow STIS slit would exclude most of the surrounding bulge starlight, enabling a more accurate measurement of the WR emission blend and a search for other, weaker WR spectral features.

In addition to the possible young stellar population, there may be an accretion-powered active nucleus in NGC 6500. If we allow for the possibility that the emission lines in NGC 6500 result from photoionization by an AGN-like continuum source, then we can estimate the UV flux that 
would be received from the central source in the absence of any obscuration. Assuming a power-law continuum with $f_{\nu} \propto \nu^{-\alpha}$, the slope $\alpha$ and the $\mathrm{H} \beta \mathrm{EW}$ are related by $\mathrm{EW}(\mathrm{H} \beta)=(560 / \alpha)(3 / 16)^{\alpha} \AA$ in the case of complete covering of the source by clouds optically thick to Lyman continuum photons (Ferland \& Netzer 1983). The ionizing continuum need not be a single power law, particularly if a "big blue bump" is present, but the spectral energy distribution of M81 shows that at least some LINERs lack this feature (Ho et al. 1996). In the more realistic case of incomplete source covering, this relation provides an upper limit to the $\mathrm{H} \beta \mathrm{EW}$. Using the $\mathrm{H} \beta$ flux from Ho et al. (1997c) and assuming $\alpha=1$, we find that the predicted continuum flux from a central AGN, if it were emitted isotropically and completely unobscured except for Galactic extinction, would be $\geq 6 \times 10^{-16} \mathrm{erg} \mathrm{s}^{-1} \mathrm{~cm}^{-2} \AA^{-1}$ at $2500 \AA$. This limit is a factor of 2.4 greater than the observed $2500 \AA$ continuum flux, indicating that if the LINER emission in NGC 6500 were solely due to nonstellar photoionization, then the central source must be heavily obscured. With the constraint that a point source makes up at most $7 \%$ of the UV continuum flux, the internal extinction toward the central continuum source would be at least $E(B-V)=0.58$ mag. Given this evidence for an "ionizing photon deficit," it is tempting to speculate that there may be a nonstellar UV continuum which is emitted anisotropically, or obscured by a dense molecular torus, as is presumed to occur in many Seyfert 2 galaxies (Wilson et al. 1988).

If NGC 6500 does contain an AGN hidden within an obscuring torus, then scattered AGN radiation could be an alternative source of the spatially extended UV continuum emission. WFPC2 UV imaging polarimetry could reveal whether scattered radiation contributes substantially to the UV continuum. One possible argument against a scattering origin for the bulk of the UV continuum emission comes from the fact that no broad lines are detected in NGC 6500. In the LINER 1 nuclei of M81 and NGC 4579, the broad C IV emission has an EW of 140 and $150 \AA$, respectively (HO et al. 1996; Barth et al. 1996b). If we suppose that the central engine of NGC 6500 is simply an obscured version of those in M81 and NGC 4579, and if the scattering geometries are the same for the continuum source and BLR, then broad C IV should be seen in the extended emission, with an $\mathrm{EW}$ of $\sim 150 \AA$ with respect to the scattered continuum. A conservative upper limit to the EW of broad C IV in NGC 6500 is $10 \AA$, where we have assumed FWHM $=4700 \mathrm{~km} \mathrm{~s}^{-1}$, the average of the broad C IV widths of NGC 4579 and M81. However, this argument rests on the assumption that the intrinsic EW of the broad-line emission in NGC 6500 is similar to those of the other LINER 1 galaxies, which need not be the case.

To summarize, we find that the UV continuum shape is reasonably well represented by a starburst of age 70-100 Myr, although older stars clearly contribute to the continuum longward of $2800 \AA$. A substantial contribution to the far-UV continuum from even younger stars cannot be ruled out, particularly given the uncertainty in the extinction correction, but we do not detect definite UV spectral features from WR or O stars. The possible He II $\lambda 4686$ feature is the only evidence for such a young population. It is conceivable that a population of WR and early O-type stars could be partly responsible for the LINER emission, although it is unlikely that hot stars alone could account for the majority of the NLR luminosity. If the NLR is photoionized by a nonstellar 
continuum, then the central source must be obscured along our line of sight; a search for ionization cones with HST narrow-band images could test this possibility. Scattered AGN radiation could contribute to the spatially extended UV continuum emission, but without polarimetric observations there is no clear way to estimate what fraction of the UV continuum might be nonstellar in origin.

\subsection{Emission-Line Diagnostics}

Examination of the UV spectrum shows that the NLR of NGC 6500 is remarkable for its lowexcitation characteristics. In contrast to low-luminosity Seyfert nuclei such as NGC 1566 Kriss et al. 1991) and NGC 4395 (Filippenko, Ho, \& Sargent 1993), the low-ionization lines of C II] $\lambda 2326$ and $\mathrm{Mg}$ II $\lambda 2800$ are among the strongest in the UV spectrum of NGC 6500, while C IV $\lambda 1549$ is undetected. The UV emission lines, with FWHM $=730-1050 \mathrm{~km} \mathrm{~s}^{-1}$, are broader than the optical lines, which have FWHM of $550-700 \mathrm{~km} \mathrm{~s}^{-1}$, but this discrepancy may be solely due to the difference in aperture sizes between the UV and optical measurements. The UV line profiles do not show broad wings, and we conclude that they arise from the NLR and that no BLR is detected.

Applying the IRAF task TEMDEN to the emission-line fluxes measured by Ho et al. (1997c), we have estimated the density and electron temperature of the NLR with standard optical line-ratio diagnostics. For the $[\mathrm{S} \mathrm{II}]$ lines, we find the ratio $I(6717) / I(6731)=1.13$, indicating a density of

$n_{e}=350 \mathrm{~cm}^{-3}$ (for $T_{e}=10^{4} \mathrm{~K}$ ) or $n_{e}=460 \mathrm{~cm}^{-3}$ (for $T_{e}=2.5 \times 10^{4} \mathrm{~K}$ ) for the [S II]-emitting region. From the [O III] $\lambda \lambda 4959,5007 / \lambda 4363$ ratio of 27.9 , we find $T_{e}=2.6 \times 10^{4}, 2.1 \times 10^{4}$, and $1.1 \times 10^{4} \mathrm{~K}$ for densities of $n_{e}=10^{2}, 10^{5}$, and $10^{6} \mathrm{~cm}^{-3}$, respectively. Such high inferred temperatures indicate that either high-density $\left(\gtrsim 10^{6} \mathrm{~cm}^{-3}\right)$ regions are present in the NLR, or, alternatively, that shock heating plays a significant role.

Photoionization models with low ionization parameter make quite different predictions from the DS fast shock models for the strengths and ratios of the UV emission lines. In the shock models, the cooling postshock region radiates strongly in lines of highly ionized species because of the high electron temperatures generated by the shock. The primary difference between LINER and Seyfert spectra, in the DS model, is that LINERs lack sufficient gas to form precursors, the preshock regions photoionized by extreme-UV and X-ray photons generated in the shock. Without such precursor regions, the shock models predict a low-excitation optical spectrum similar to those of LINERs, and a high-excitation UV spectrum in which lines such as C IV $\lambda 1549$ and N V $\lambda 1240$ are important coolants. On the other hand, in standard photoionization models with $U \approx 10^{-3.5}-10^{-4.0}$, which roughly reproduce the optical emission-line spectra of LINERs, lines of highly ionized species such as C IV and N V are extremely weak or totally absent because of the low ionization parameter. Thus, the strength of the high-excitation UV lines is an important diagnostic that can help to discriminate between the two classes of models.

To investigate the ionization level of the NLR gas, we compare the relative strengths of the UV lines of carbon: C II] $\lambda 2326, \mathrm{C}$ III] $\lambda 1909$, and C IV $\lambda 1549$. In Figure 4 we plot the measured 
ratio (C III]/C IV) against (C II]/C III]) and the values predicted by various models. Three sets of model results are included; we describe the basic model parameters here, and refer the reader to the sources listed below for full details on the model calculations. For the DS fast shock calculations, a preshock density of $n(\mathrm{H})=1 \mathrm{~cm}^{-3}$ is assumed, the shock velocity is in the range $150-500 \mathrm{~km} \mathrm{~s}^{-1}$, and model results are plotted for two values of the "magnetic parameter" $B / n^{1 / 2}$, which controls the compression of the postshock gas. For slower shocks, we include the results of models $\mathrm{C}-\mathrm{G}$ from Raymond (1979), with velocities of $70-140 \mathrm{~km} \mathrm{~s}^{-1}$ and preshock density of $n(\mathrm{H})=10 \mathrm{~cm}^{-3}$. The photoionization model results are adapted from calculations performed by Ho et al. (1993) using the photoionization code CLOUDY (Ferland 1991). These calculations were done for a single slab of gas illuminated by a power-law continuum with $f_{\nu} \propto \nu^{-1.5}$, and we display the results for densities ranging from $n_{e}=10^{2.5}$ to $10^{6.0} \mathrm{~cm}^{-3}$ and ionization parameters ranging from $\log U=-2.5$ to -3.5. All of the models used an undepleted solar abundance set (e.g., Grevesse \& Anders 1989), except that $\mathrm{Ca}$ and Fe were each depleted by 0.2 dex in the DS calculations.

The differences between the DS shock model predictions and photoionization calculations are readily apparent in this diagram, as the DS models all predict C IV > C III], while all photoionization models with $\log U \leq-2.5$ have $\mathrm{C} \mathrm{III]}>\mathrm{C}$ IV. In the DS models, the $\mathrm{C} \mathrm{III]/C} \mathrm{IV} \mathrm{ratio}$ ranges from 0.1 to 0.9 , but even in the most favorable case of $500 \mathrm{~km} \mathrm{~s}^{-1}$ shocks with high magnetic parameter, the predicted ratio is a factor of 4.5 too small to be consistent with the observed limit. Other line ratios are in conflict with the data as well: the fast shock models predict that the $\mathrm{N} \mathrm{V}$ $\lambda 1240$ and O IV $\lambda 1402$ lines should be comparable to or stronger than C III], and that for low magnetic parameter and low shock velocities $\left(\leq 200 \mathrm{~km} \mathrm{~s}^{-1}\right)$, O III] $\lambda 1666$ should be comparable to or stronger than $\mathrm{C} \mathrm{III]} \mathrm{and} \mathrm{C} \mathrm{II].} \mathrm{The} \mathrm{predictions} \mathrm{of} \mathrm{the} \mathrm{DS} \mathrm{fast} \mathrm{shock} \mathrm{models} \mathrm{are} \mathrm{clearly} \mathrm{at}$ odds with the low-ionization state of the NGC 6500 NLR. It is worth noting as well that the UV emission-line ratios in NGC 6500 are quite unlike those seen in supernova remnants. Spectra of shock-excited filaments in the Cygnus Loop and of Puppis A with shock velocities of $\sim 170 \mathrm{~km} \mathrm{~s}^{-1}$ show C IV > He II (Blair et al. 1991; Blair et al. 1995), while in NGC 6500 our upper limit on C IV is a factor of 2 weaker than the strength of He II.

Slower shocks, on the other hand, might provide a better match to the observed line ratios. The Raymond (1979) calculations span a wide range in the C III]/C IV ratio, and at the lowest velocities $(<100 \mathrm{kms})$ are consistent with the $\mathrm{C} \mathrm{III]/C} \mathrm{IV} \mathrm{limit} \mathrm{and} \mathrm{nearly} \mathrm{consistent} \mathrm{with} \mathrm{the}$ $\mathrm{C}$ II]/C III] ratio in NGC 6500. These models cannot fully describe the NLR conditions within NGC 6500 , though, because they underpredict [O I] $\lambda 6300$ by an order of magnitude, yielding a spectrum that does not meet the definition of a LINER. However, given the tremendous parameter space available for shock models, it seems likely that some combination of shock parameters, or some superposition of different shock models, could adequately reproduce the range of emission-line strengths observed in NGC 6500. Certainly, shocks must be occurring to some extent within the nucleus of NGC 6500, given the kinematic and morphological evidence for a nuclear outflow.

The lack of C IV $\lambda 1549$ emission in NGC 6500 is consistent with the predictions of photoionization calculations as long as $\log U \lesssim-2.5$, and the $\mathrm{C}$ II]/C III] ratio suggests $\log U=-3.0$. 
This result is inconsistent with the optical spectrum, however, as the optical line ratios [O II] $\lambda \lambda 3726,3729 /[\mathrm{O}$ III] $\lambda 5007$ and [O I] $\lambda 6300 /[\mathrm{O}$ III] $\lambda 5007$ in NGC 6500 are better fit by the models with $\log U=-3.5$ to -4.0 . The mismatch indicates that a range of values of $U$ must be present if the NLR gas is photoionized. The high [O III] temperature also suggests that the NLR of NGC 6500 may be stratified in density (Filippenko \& Halpern 1984), and it is clear that both density and ionization stratification must be taken into account when constructing realistic photoionization models for LINERs. As in the case of NGC 4579 (Barth et al. 1996b), the power-law photoionization models overpredict the strength of He II $\lambda 1640$ (relative to C III]) for densities less than $10^{6} \mathrm{~cm}^{-3}$, and the discrepancy is more severe if part or all of the $\lambda 1640$ line is generated in WR atmospheres. The weak He II line relative to photoionization predictions is a likely indication that the ionizing continuum falls off steeply at energies higher than $54.4 \mathrm{eV}$ (e.g., Péquignot 1984). Alternately, the weakness of He II in LINERs could result from a composite cloud population consisting of optically thin and optically thick components, as in the scenario of Binette et al. (1996).

It would be premature, however, to conclude that the NLR of NGC 6500 is entirely photoionized by an AGN, and it is not clear that this ambiguity can be resolved with the present data, especially in view of the overlap in Figure 4 between the photoionization models and the slow shock models. If the spatially extended emission arises from gas illuminated by a central source, then the roughly constant optical emission-line ratios suggest that the ionization parameter does not vary strongly with radius, which would imply that $n_{e} \propto r^{-2}$ (Filippenko 1984). However, the spatially extended low-ionization emission may also be suggestive of shock excitation, or possibly of a spatially distributed source of ionizing photons. In addition, it is well known that the extended emission in superwind galaxies can have a LINER-like optical spectrum (Heckman et al. 1987). Given the evidence for a nuclear outflow in NGC 6500, it seems at least plausible that the extended NLR emission could be generated by shocks in the expanding wind, while the nuclear emission might be due to gas photoionized by a hidden AGN. Long-slit spectra taken with STIS could be used to search for spatial gradients in the emission-line ratios that might indicate a transition from photoionization at the nucleus to shock excitation in the extended emission. A comparison of the UV emission-line spectrum with the predictions of composite shock plus photoionization models (e.g., Contini 1997) would be instructive. As discussed in $\S 3.1$, hot stars at the nucleus may also contribute a sizeable fraction of the ionizing photon luminosity. Deep STIS UV spectra of NGC 6500 would also enable a more sensitive search for C IV emission and a better assessment of the importance of hot stars in this object, as well as an independent check on the reality of the possible $\mathrm{N}$ V emission and C IV absorption.

At a more fundamental level, the key question that must be addressed is whether LINER 2 nuclei are powered by accretion onto a supermassive black hole, or by massive stars, or by some other process. The ionization state of the NLR gas provides clues but does not yield a compelling answer to this question. Is there any evidence that requires an AGN to be present in the nucleus of NGC 6500? Broad emission lines, one of the clearest signs of such activity, are conspicuously absent from the UV spectrum, and we can only speculate as to whether they may be hidden behind 
obscuring material or whether they are not present at all in NGC 6500. If a compact UV continuum source is present and luminous enough to contribute significantly to the NLR ionization, then it must be highly obscured. Optical spectropolarimetry could reveal hidden broad-line emission and continuum polarization, and a hard X-ray continuum might be detectable with AXAF observations. At present, the compact, flat-spectrum radio source is perhaps the most direct indication of a hidden AGN in this galaxy. As optical and UV studies have been inconclusive, future radio and X-ray observations may provide the best means for determining the nature of this object.

\section{Summary and Conclusions}

Optical studies of LINERs generally focus on the problem of determining whether shock heating or photoionization is the dominant ionization mechanism within the NLR. In NGC 6500, we have an example of a LINER in which shocks, photoionization by an AGN, and ionization by hot stars may all make energetically significant contributions, and we hope to reach an understanding of the relationships between the nuclear starburst, the outflow, and the hidden active nucleus that may be present. The available data do not suffice to disentangle these components from one another, but our study of the UV spectrum of NGC 6500 does lead to the following conclusions:

1. If the UV continuum is due to starlight, then stars of age $\lesssim 100 \mathrm{Myr}$ are present in the nucleus. The He II $\lambda 4686$ emission line, which we tentatively attribute to WR stars, indicates that the region may have experienced a burst of star formation within the last several Myr. No clear signatures of such young stars appear in the UV spectrum, but data of higher $\mathrm{S} / \mathrm{N}$ may reveal the P Cygni features of C IV and Si IV which are expected from such a young population. Rough orderof-magnitude estimates indicate that this young burst population may contribute substantially to the ionization of the NLR, but better data are needed to fully assess this possibility.

2. If the NLR of NGC 6500 is photoionized by an AGN, then the central continuum source must be highly obscured along our line of sight but visible to the NLR. Future polarization measurements can test whether scattered radiation from a hidden AGN contributes to the spatially extended UV continuum emission.

3. The UV emission-line ratios are incompatible with the predictions of the Dopita \& Sutherland $(1995,1996)$ fast shock models, which predict strong lines of C IV, O IV, and other highly

ionized species. Slower $\left(\lesssim 100 \mathrm{~km} \mathrm{~s}^{-1}\right)$ shocks can match the UV emission-line ratios more successfully, as can power-law photoionization models.

Although the status of NGC 6500 remains somewhat ambiguous, the UV spectra of the LINERs M81, NGC 4579, and NGC 6500 provide ample evidence to contradict the claim of DS that the narrow-line regions of all Seyferts and LINERs are primarily excited by fast shocks. Other objects, such as the nuclear disk of M87 (Dopita et al. 1996), may well be excited by fast shocks, but there are not yet any examples known of typical LINER nuclei having high-excitation UV emission-line spectra. Future STIS observations should provide far better data with which to assess the relative 
contributions of shocks, hot stars, and nonstellar continuum sources to the ionization of LINERs.

We are grateful to Mike Eracleous, Nancy Levenson, Daniel Stern, Jules Halpern, and John Raymond for helpful advice, and to an anonymous referee for very constructive suggestions that improved this paper. We also owe thanks to Gary Ferland, and to Gustavo Bruzual and Stéphane Charlot, for making their excellent software (CLOUDY and GISSEL96, respectively) available to the community. This research has made use of the NASA/IPAC Extragalactic Database (NED),

which is operated by the Jet Propulsion Laboratory, California Institute of Technology, under contract with NASA. Financial support was provided by grants GO-5431-93A, AR-5291-93A, AR5792-94A, and GO-6112-94A from the Space Telescope Science Institute, which is operated by AURA, Inc., under contract with NASA.

\section{REFERENCES}

Barth, A. J., Ho, L. C., Filippenko, A. V., \& Sargent, W. L. W. 1996a, in The Physics of LINERs in View of Recent Observations, ed. M. Eracleous et al. (San Francisco: ASP), 153

Barth, A. J., Reichert, G. A., Filippenko, A. V., Ho, L. C., Shields, J. C., Mushotzky, R. F., \& Puchnarewicz, E. M. 1996b, AJ, 112, 1829

Binette, L., Wilson, A. S., \& Storchi-Bergmann, T. 1996, A\&A, 312, 365

Blair, W. P., et al. 1991, ApJ, 379, L33.

Blair, W. P., Raymond, J. C., Long, K. S., \& Kriss, G. A. 1995, ApJ, 454, L35.

Bruzual A., G., \& Charlot, S. 1993, ApJ, 405, 538

Burstein, D., \& Heiles, C. 1984, ApJS, 54, 33

Calzetti, D., Kinney, A. L., \& Storchi-Bergmann, T. 1994, ApJ, 429, 582

Cardelli, J. A., Clayton, G. C., \& Mathis, J. S. 1989, ApJ, 345, 245

Contini, M. 1997, A\&A, 323, 71

Crane, P. C. 1979, AJ, 84, 281

David, L. P., Harnden, F. R., Kearns, K. E., \& Zombeck, M. V. 1996, The ROSAT HRI Calibration Report, U.S. ROSAT Science Data Center document

Dopita, M. A., \& Sutherland, R. S. 1995, ApJ, 455, 468 (DS)

Dopita, M. A., \& Sutherland, R. S. 1996, ApJS, 102, 161 (DS) 
Dopita, M. A., et al. 1996, in The Physics of LINERs in View of Recent Observations, ed. M. Eracleous et al. (San Francisco: ASP), 44

Fabbiano, G. 1996, in The Physics of LINERs in View of Recent Observations, ed. M. Eracleous et al. (San Francisco: ASP), 56

Fanelli, M. N., O'Connell, R. W., Burstein, D., \& Wu, C.-C. 1990, ApJ, 364, 272

Ferland, G. J. 1991, Ohio State University Internal Report 91-01 (Columbus: OSU Astronomy)

Ferland, G. J., \& Netzer, H. 1983, ApJ, 264, 105

Filippenko, A. V. 1984, Ph.D. Thesis, Caltech (Ann Arbor: University Microfilms)

Filippenko, A. V. 1996, in The Physics of LINERs in View of Recent Observations, ed. M. Eracleous et al. (San Francisco: ASP), 17

Filippenko, A. V,. \& Halpern, J. P. 1984, ApJ, 285, 458

Filippenko, A. V., Ho, L. C., \& Sargent, W. L. W. 1993, ApJ, 410, L75

Filippenko, A. V., \& Sargent, W. L. W. 1985, ApJS, 57, 503

Filippenko, A. V., \& Terlevich, R. 1992, ApJ, 397, L79

González Delgado, R. M., \& Pérez, E. 1996, MNRAS, 281, 1105

Grevesse, N., \& Anders, E. 1989, in Cosmic Abundances of Matter, ed. C. J. Washington (New York: AIP), 1

Heckman, T. M. 1980, A\&A, 87, 152

Heckman, T. M., Armus, L., \& Miley, G. K. 1987, AJ, 92, 276

Heckman, T. M., Armus, L., \& Miley, G. K. 1990, ApJS, 74, 833

Ho, L. C., Filippenko, A. V., \& Sargent, W. L. W. 1993, ApJ, 417, 63

Ho, L. C., Filippenko, A. V., \& Sargent, W. L. W. 1996, ApJ, 462, 183

Ho, L. C., Filippenko, A. V., \& Sargent, W. L. W. 1997a, ApJS, in press

Ho, L. C., Filippenko, A. V., Sargent, W. L. W., \& Peng, C. Y. 1997b, ApJS, in press

Ho, L. C., Filippenko, A. V., \& Sargent, W. L. W. 1997c, ApJS, in press

Jones, D. L., Sramek, R. A., \& Terzian, Y. 1981, ApJ, 246, 28

Koratkar, A., \& Martin, S. 1995, FOS Instrument Science Report CAL/FOS-145 (Baltimore: STScI) 
Kriss, G. A., Hartig, G. F., Armus, L., Blair, W. P., Caganoff, S., \& Dressel, L. 1991, ApJ, 377, L13

Leitherer, C., \& Heckman, T. M. 1995, ApJS, 96, 9

Leitherer, C., Robert, C., \& Heckman, T. M. 1995, ApJS, 99, 173

Leitherer, C., Vacca, W. D., Conti, P. S., Filippenko, A. V., Robert, C., \& Sargent, W. L. W. 1996, ApJ, 465, 717

Mathis, J. S. 1990, ARA\&A, 28, 37

Morse, J. A., Raymond, J. C., \& Wilson, A. S. 1996, PASP, 108, 426

Murphy, E. M., Lockman, F. J., Laor, A., \& Elvis, M. 1996, ApJS, 105, 369

Péquignot, D. 1984, A\&A, 131, 159

Pogge, R. W. 1989, ApJS, 71, 433

Raymond, J. C. 1979, ApJS, 39, 1

Rosa, M. R. 1994, FOS Instrument Science Report CAL/FOS-127 (Baltimore: STScI)

Salpeter, E. E. 1955, ApJ, 121, 161

Serlemitsos, P., Ptak, A., \& Yaqoob, T. 1996, in The Physics of LINERs in View of Recent Observations, ed. M. Eracleous et al. (San Francisco: ASP), 56

Shields, J. C. 1992, ApJ, 399, L27

Unger, S. W., Pedlar, A., \& Hummel, E. 1989, A\&A, 208, 14

Vacca, W. D., \& Conti, P. S. 1992, ApJ, 401, 543

Wilson, A. S., Ward, M. J., \& Haniff, C. A. 1988, ApJ, 334, 121 
Table 1. Ultraviolet Emission Lines in NGC 6500.

\begin{tabular}{lcccc}
\hline \hline Line & $\begin{array}{c}F \times 10^{15} \\
\left(\mathrm{erg} \mathrm{cm}^{-2} \mathrm{~s}^{-1}\right)\end{array}$ & $\begin{array}{c}I \times 10^{15} \\
\left(\mathrm{erg} \mathrm{cm}^{-2} \mathrm{~s}^{-1}\right)\end{array}$ & $\begin{array}{c}\text { FWHM } \\
\left(\mathrm{km} \mathrm{s}^{-1}\right)\end{array}$ & Notes \\
\hline Ly $\lambda 1216$ & 29.0 & 146 & 730 & $\mathrm{a} ?$ \\
N V $\lambda 1240 ?$ & $<1.2$ & $<5.7$ & $\ldots$ & $\mathrm{b}, \mathrm{c} ?$ \\
C IV $\lambda 1549$ & $<1.0$ & $<3.3$ & $\ldots$ & \\
He II $\lambda 1640$ & 1.9 & 6.1 & $\ldots$ & \\
Si III] $\lambda 1883$ & 1.1 & 3.6 & $\ldots$ & \\
C III] $\lambda 1909$ & 4.0 & 13.4 & 1050 & \\
C II] $\lambda 2326$ & 4.1 & 14.7 & 820 & \\
[O II] $\lambda 2470$ & 0.7 & 2.1 & $\ldots$ & $\mathrm{c}$ \\
Mg II $\lambda 2800$ & 4.5 & 11.0 & $\ldots$ & $\mathrm{a}$ \\
\hline
\end{tabular}

Note. $-F=$ measured flux; $I=$ flux corrected for total reddening of $E(B-V)=0.16$ mag. Extinction correction factors were computed using the extinction curve of Cardelli et al. (1989). (a) Affected by absorption. (b) Line identification uncertain. (c) Line contaminated by noisy diode. 

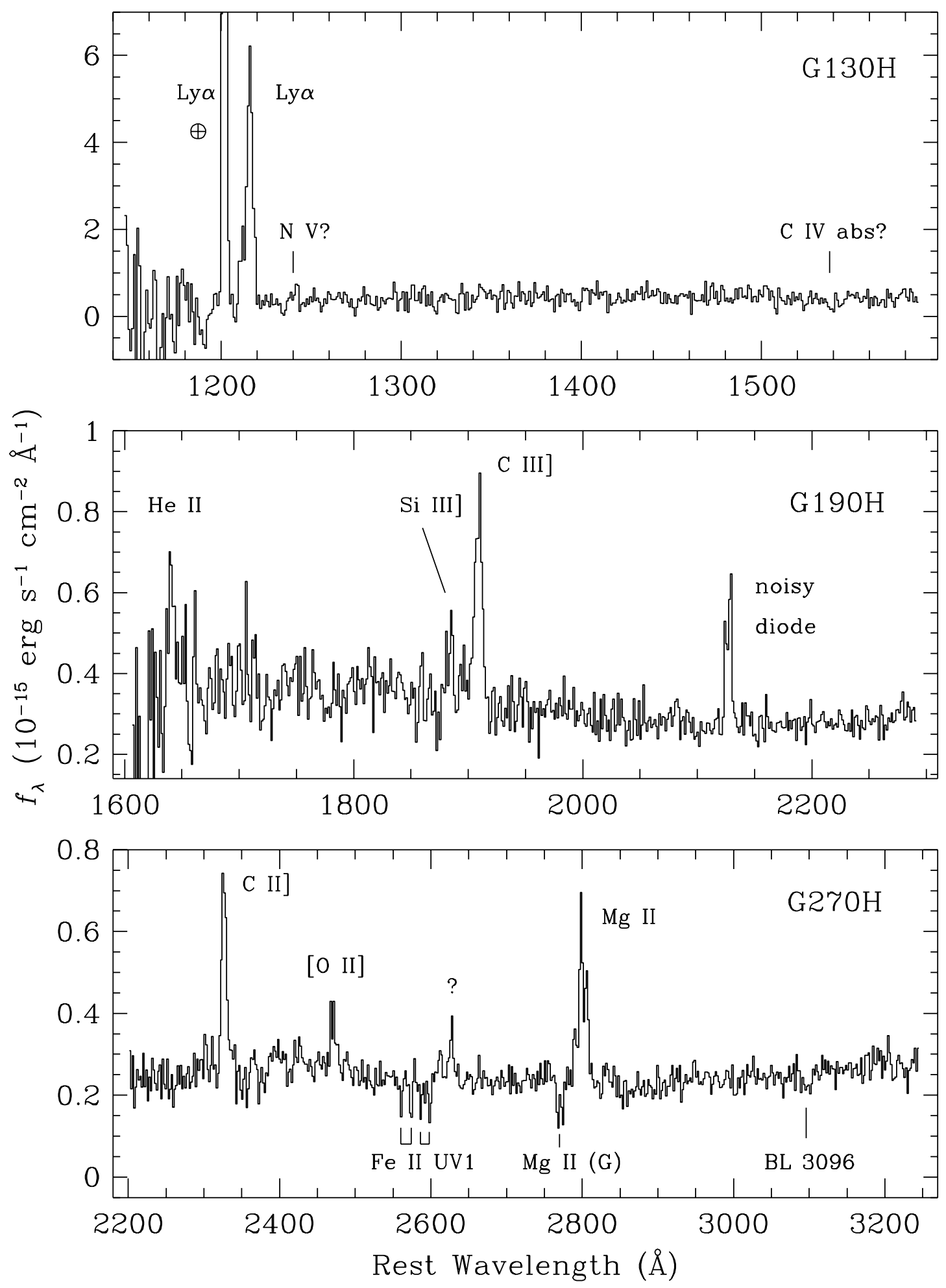

Fig. 1.- Ultraviolet spectrum of NGC 6500. Emission and absorption features discussed in the text are labelled. The absorption lines labelled as being members of the Fe II UV1 multiplet include the Galactic and the internal lines at rest wavelengths of 2586 and $2599 \AA$. Internal absorption due to Mg II is seen superposed on the profile of the Mg II emission line. 


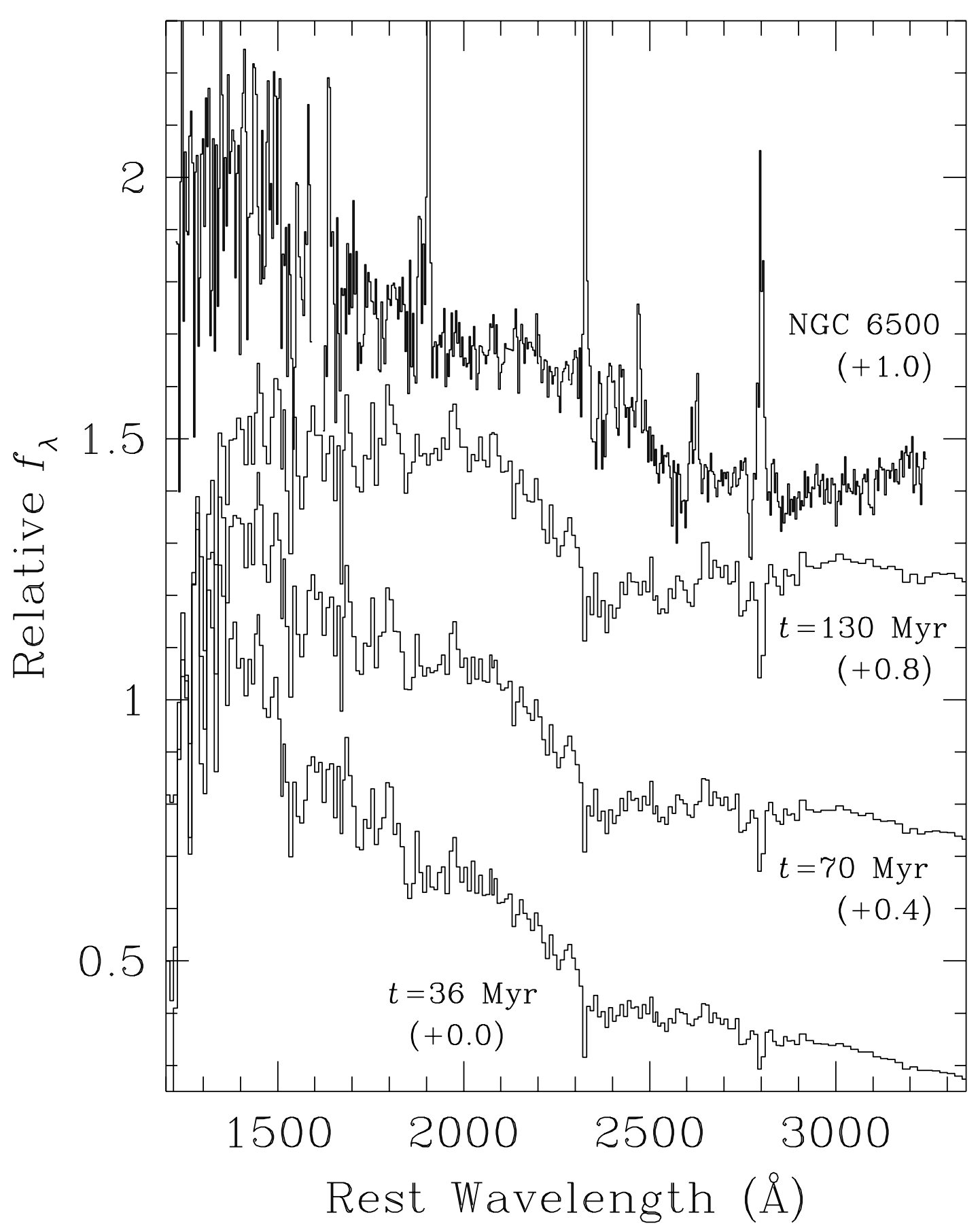

Fig. 2.- Comparison of the UV continuum shape of NGC 6500 with synthetic spectra of singleburst evolutionary models, for ages of 36, 70, and 130 Myr. The NGC 6500 continuum has been corrected for a Galactic extinction of $E(B-V)=0.09 \mathrm{mag}$, and the spectrum has been binned for clarity to $2 \AA \mathrm{bin}^{-1}$ above $1600 \AA$ and to $4 \AA \mathrm{bin}^{-1}$ below $1600 \AA$. All spectra have been scaled to a common flux at $2000 \AA$. 


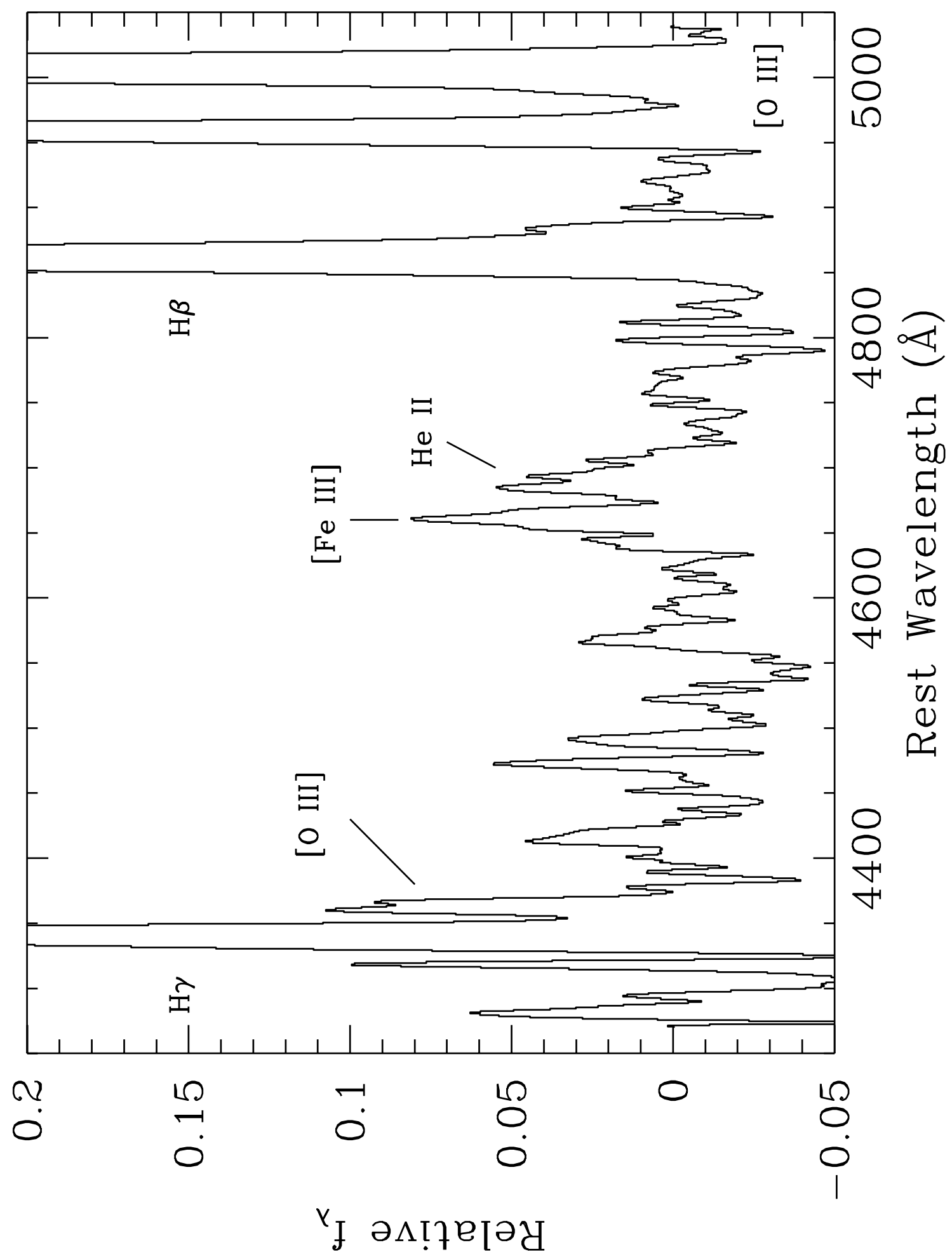

Fig. 3.- Starlight-subtracted optical spectrum of NGC 6500, adapted from Ho et al. (1997c). A "bump," part of which may originate from Wolf-Rayet stars, is present at 4600-4700 $\AA$. 


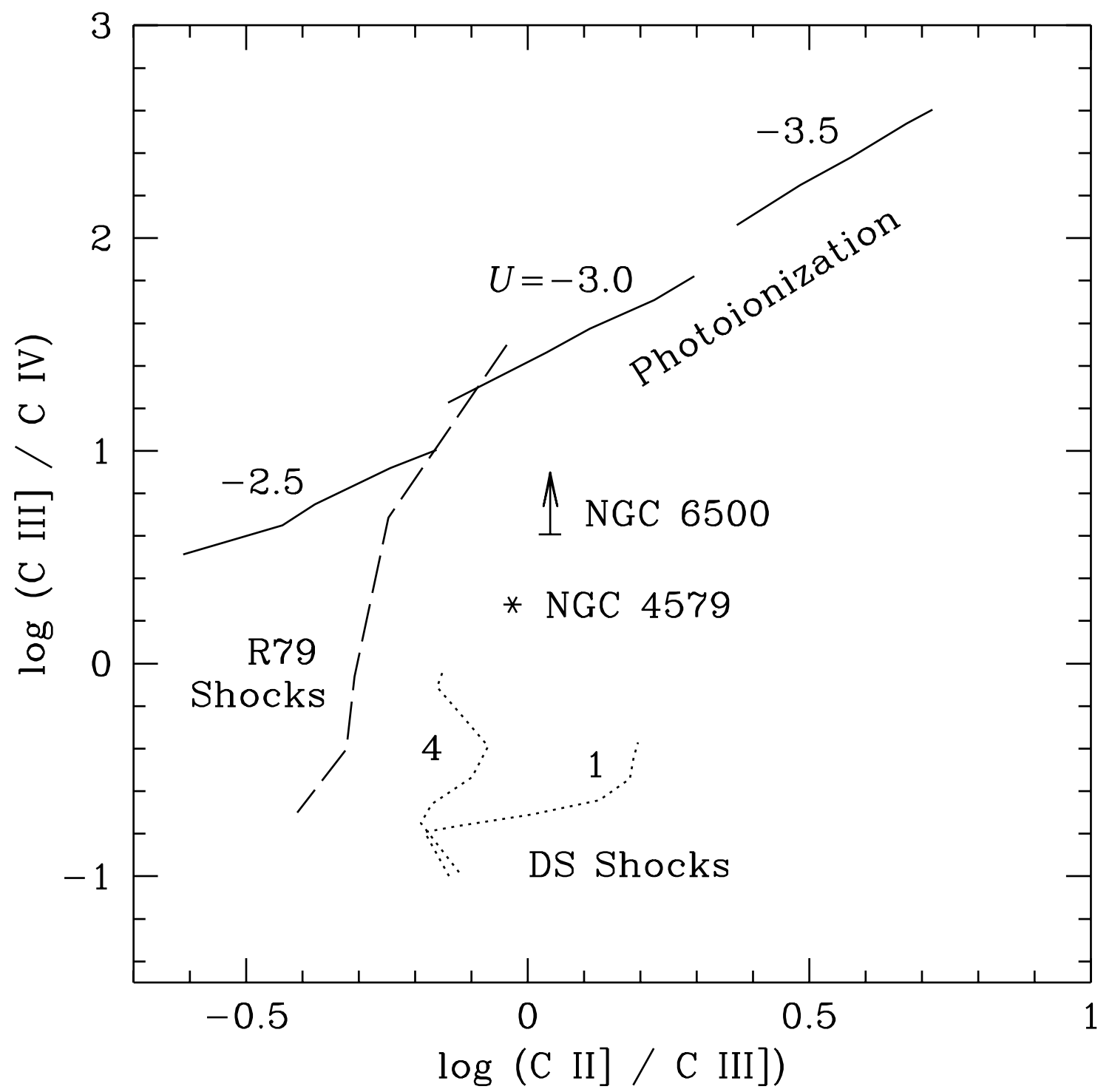

Fig. 4.- Diagnostic diagram for UV emission lines of carbon: C II] $\lambda 2326$, C III] $\lambda 1909$, and C IV $\lambda 1549$. Solid lines: Power-law photoionization models computed by Ho et al. (1993), using CLOUDY, for $f_{\nu} \propto \nu^{-1.5}$ and $\log U=-2.5,-3.0$, and -3.5 . Along each curve, density increases from $n_{e}=10^{2.5} \mathrm{~cm}^{-3}$ to $10^{6} \mathrm{~cm}^{-3}$, with highest densities at the lower left of each curve. Dotted lines: Shock models of Dopita \& Sutherland (1996), for magnetic parameter $B / n^{1 / 2}=1$ and $4 \mu \mathrm{G}$ $\mathrm{cm}^{3 / 2}$. Shock velocity ranges from 150 to $500 \mathrm{~km} \mathrm{~s}^{-1}$, with fastest shocks at the top of each curve. Long-dashed line: Shock models C through G from Raymond (1979), with velocities ranging from $70.7 \mathrm{~km} \mathrm{~s}^{-1}$ (top) to $141 \mathrm{~km} \mathrm{~s}^{-1}$ (bottom). For NGC 4579, only the narrow-line component is included. 